\title{
Do Halo Nuclei Follow Rutherford Elastic Scattering at Energies Below the Barrier? The Case of ${ }^{11} \mathrm{Li}$
}

\author{
M. Cubero, ${ }^{1,2}$ J. P. Fernández-García, ${ }^{3,4}$ M. Rodríguez-Gallardo, ${ }^{3}$ L. Acosta, ${ }^{5}$ M. Alcorta, ${ }^{1}$ M. A. G. Alvarez, ${ }^{3,4}$ \\ M. J. G. Borge, ${ }^{6, *}$ L. Buchmann, ${ }^{7}$ C. A. Diget, ${ }^{8}$ H. Al Falou, ${ }^{9}$ B. R. Fulton, ${ }^{8}$ H. O. U. Fynbo, ${ }^{10}$ D. Galaviz, ${ }^{11}$ \\ J. Gómez-Camacho, ${ }^{3,4}$ R. Kanungo, ${ }^{9}$ J. A. Lay, ${ }^{3}$ M. Madurga, ${ }^{6}$ I. Martel, ${ }^{5}$ A. M. Moro, ${ }^{3}$ I. Mukha, ${ }^{4}$ T. Nilsson, ${ }^{12}$ \\ A. M. Sánchez-Benítez, ${ }^{5}$ A. Shotter, ${ }^{7,13}$ O. Tengblad, ${ }^{6}$ and P. Walden ${ }^{7}$ \\ ${ }^{1}$ Instituto de Estructura de la Materia CSIC, E28006 Madrid, Spain \\ ${ }^{2}$ CICANUM, Universidad de Costa Rica UCR, Apartado 2060 San José, Costa Rica \\ ${ }^{3}$ Departamento de FAMN, Universidad de Sevilla, 41080 Seville, Spain \\ ${ }^{4}$ Centro Nacional de Aceleradores, Universidad de Sevilla/Junta de Andalucía/CSIC, 41092 Seville, Spain \\ ${ }^{5}$ Departamento de Física Aplicada, Universidad de Huelva, 21071 Huelva, Spain \\ ${ }^{6}$ Instituto de Estructura de la Materia CSIC, E28006 Madrid, Spain \\ ${ }^{7}$ TRIUMF, V6T2A3 Vancouver, British Columbia, Canada \\ ${ }^{8}$ Department of Physics, University of York, YO 10 5DD Heslington, York, United Kingdom \\ ${ }^{9}$ Department of Astronomy and Physics, Saint Mary's University, Halifax B3H3C3, Nova Scotia, Canada \\ ${ }^{10}$ Department of Physics and Astronomy, Aarhus University, DK-8000 Aarhus, Denmark \\ ${ }^{11}$ CFNUL, Universidade de Lisboa, 1649-003 Lisbon, Portugal \\ ${ }^{12}$ Fundamental Physics, Chalmers University of Technology, 41296 Göteborg, Sweden \\ ${ }^{13}$ School of Physics and Astronomy, University of Edinburgh, EH9 3JZ, Edinburgh, United Kingdom
}

(Received 7 October 2012; published 26 December 2012)

The first measurement of the elastic scattering of the halo nucleus ${ }^{11} \mathrm{Li}$ and its core ${ }^{9} \mathrm{Li}$ on ${ }^{208} \mathrm{~Pb}$ at energies near the Coulomb barrier is presented. The ${ }^{11} \mathrm{Li}+{ }^{208} \mathrm{~Pb}$ elastic scattering shows a strong reduction with respect to the Rutherford cross section, even at energies well below the barrier and down to very small scattering angles. This drastic change of the elastic differential cross section observed in ${ }^{11} \mathrm{Li}+$ ${ }^{208} \mathrm{~Pb}$ is the consequence of the halo structure of ${ }^{11} \mathrm{Li}$, as it is not observed in the elastic scattering of its core ${ }^{9} \mathrm{Li}$ at the same energies. Four-body continuum-discretized coupled-channels calculations, based on a three-body model of the ${ }^{11} \mathrm{Li}$ projectile, are found to explain the measured angular distributions and confirm that the observed reduction is mainly due to the strong Coulomb coupling to the dipole states in the low-lying continuum of ${ }^{11} \mathrm{Li}$. These calculations suggest the presence of a low-lying dipole resonance in ${ }^{11} \mathrm{Li}$ close to the breakup threshold.

DOI: 10.1103/PhysRevLett.109.262701

PACS numbers: 25.60.Bx, 25.60.Gc, 25.60.- $\mathrm{t}$

One century ago, Rutherford [1] inferred the structure of the atom from the reaction data measured by Geiger and Marsden [2]. Since then, nuclear structure properties have often been deduced from nuclear reaction studies. With the advent of the first accelerated radioactive beams, new nuclear structures were discovered, such as the existence of a halo in some very loosely bound nuclei. In fact, 25 years ago, Hansen and Jonson [3] interpreted the large interaction cross section observed in ${ }^{11} \mathrm{Li}$ with light targets by Tanihata et al. [4] as due to the high probability of the outermost nucleons to be at large distances from the central core, which they referred to as a halo structure. The halo structure is a threshold phenomenon due to the low binding energy of the last nucleons. Halo nuclei have several features in common, such as a rather compact core, an extended neutron distribution, and very few, if any, excited states.

The discovery of halo nuclei brought renewed interest in the modeling of nuclear reactions. This peculiar structure should affect the reaction properties at near-Coulomb barrier energies. Due to their low binding energy, the description of reactions involving halo nuclei should incorporate the coupling into the continuum. Current approaches to reaction theory involve different approximations whose validity needs to be checked when applied to halo nuclei.

The most neutron-rich bound lithium isotope, ${ }^{11} \mathrm{Li}$, is a fascinating case. Predicted to be unbound, it was identified in 1966 [5] and it is the archetype of a Borromean halo nucleus; i.e., the two different binary subsystems, ${ }^{9} \mathrm{Li}-n$ and $n-n$, are unbound, whereas the three-body system is bound by $S_{2 n}=369.15 \pm 0.65 \mathrm{keV}$ [6]. The ground state density distribution of ${ }^{11} \mathrm{Li}$ extends well beyond its core; i.e., the rms matter radius for the ${ }^{9} \mathrm{Li}$ isotope is $2.44 \pm 0.06 \mathrm{fm}$ [7], while for ${ }^{11} \mathrm{Li}$ reported values are one fermi larger. No bound excited states are known, but several resonances at $1.1 \mathrm{MeV}(\Gamma \approx 0.5 \mathrm{MeV})$ and $2.5 \mathrm{MeV}(\Gamma \approx 1.5 \mathrm{MeV})$ have been identified in different reaction studies with light ions (see Ref. [8] for a recent review). Theoretical models predict also different low-lying resonances with $J_{n n}^{\pi}=0^{+}$ and $1^{-}$[9-13], but their existence and precise location have not been clearly established experimentally.

Due to the loosely bound structure, the neutron halo is easily polarizable in the strong electric field of a heavy 
target such as ${ }^{208} \mathrm{~Pb}$. In contrast to normal nuclei, where the $E 1$ response is dominated by the giant dipole resonance, large soft electric dipole $(E 1)$ strength close to the breakup threshold has been observed in halo nuclei. The strongest $E 1$ transition observed at low excitation energy was determined in an exclusive measurement of the Coulomb dissociation of ${ }^{11} \mathrm{Li}$ at $770 \mathrm{MeV}$ at RIKEN [14]. This large E1 strength will induce a strong dynamic polarization effect of the ${ }^{11} \mathrm{Li}$ projectile when scattered with a heavy target, giving rise to long-range Coulomb couplings. According to Ref. [15], this effect should manifest itself at Coulomb barrier energies as a departure from the elastic Rutherford scattering.

In order to disentangle the contribution of the loosely bound structure of ${ }^{11} \mathrm{Li}$ from the reaction process, one should know the behavior of the core, ${ }^{9} \mathrm{Li}$, under the same conditions. No data exist for the scattering of ${ }^{11} \mathrm{Li}$ near the Coulomb barrier, and even for ${ }^{9} \mathrm{Li}$ the scattering data are scarce. The cross section of ${ }^{9} \mathrm{Li}$ on the ${ }^{208} \mathrm{~Pb}$ target was measured at $86 \mathrm{MeV}$ [16], but this energy is three times the Coulomb barrier. Near the Coulomb barrier, the fusion cross section of ${ }^{9} \mathrm{Li}+{ }^{208} \mathrm{~Pb}$ was measured at centerof-mass (c.m.) energies from 23.9 to $43.0 \mathrm{MeV}$ [17]. However, the elastic cross section was not reported.

This Letter reports on the first measurement of the scattering of the halo nucleus ${ }^{11} \mathrm{Li}$ on ${ }^{208} \mathrm{~Pb}$ at incident energies of 24.3 and $29.8 \mathrm{MeV}$, which are, respectively, below and around the Coulomb barrier $\left(V_{b} \approx 28 \mathrm{MeV}\right)$. To characterize the behavior of the core, the ${ }^{9} \mathrm{Li}+{ }^{208} \mathrm{~Pb}$ scattering has also been measured with the same setup and at the same c.m. energies of 23.1 and $28.3 \mathrm{MeV}$. The ${ }^{11} \mathrm{Li}$ elastic scattering data show a stronger reduction of the cross section than predicted in Ref. [15] for this reaction. These data have been compared with four-body continuumdiscretized coupled-channels (CDCC) calculations, based on a three-body description of the ${ }^{11} \mathrm{Li}$ nucleus, which takes into account, in addition to the dipole Coulomb couplings, other Coulomb multipoles and nuclear couplings to all orders.

The experiment was performed in the postaccelerated ISAC-II line at the TRIUMF facility (Vancouver, Canada). A primary $500 \mathrm{MeV} 100 \mu \mathrm{A}$ proton beam produced at the TRIUMF cyclotron irradiated a Ta primary target. The secondary beams of $\mathrm{Li}$ isotopes were transported to the ISAC-II facility for postacceleration [18]. The average intensity of the ${ }^{11} \mathrm{Li}$ beam, as detected in our monitor detector located $280 \mathrm{~mm}$ downstream of the $\mathrm{Pb}$ target, was $4300{ }^{11} \mathrm{Li} / \mathrm{s}$.

The accelerated incoming beam impinged on a ${ }^{208} \mathrm{~Pb}$ target tilted $75^{\circ}$ with respect to the beam direction. For the ${ }^{9} \mathrm{Li}$ study, two ${ }^{208} \mathrm{~Pb}$ targets with different thicknesses 1.45 and $1.9 \mathrm{mg} / \mathrm{cm}^{2}$ were used. Only the $1.45 \mathrm{mg} / \mathrm{cm}^{2}$ ${ }^{208} \mathrm{~Pb}$ target was bombarded by the ${ }^{11} \mathrm{Li}$ beam, in order to minimize the loss of energy resolution due to straggling in the target.
The experimental setup consisted of four telescopes, $T 1-T 4$. Two of them, $T 1$ and $T 2$, were placed in the target forward direction, each one consisting of a windowless $40 \mu \mathrm{m}$ thick double-sided silicon $(16 \times 16)$ strip detector (DSSSD) [19] acting as a $\Delta E$ detector and a $500 \mu \mathrm{m}$ thick Si PAD as an $E$ detector. Telescopes $T 3$ and $T 4$ were placed in the target backward direction, and each one consisted of a $20 \mu \mathrm{m}$ thick $\Delta E$ single-sided silicon (16) strip detector and a $60 \mu \mathrm{m}$ thick DSSSD $(16 \times 16)$ behind.

The segmentation of the detector system gives information of 256 pixels per telescope either by matching front and back strips of the DSSSD for telescopes 1 and 2 or front and back detectors in telescopes 3 and 4 . This configuration permitted a high angular resolution $\left(2^{\circ}-3^{\circ}\right.$, depending upon the detector) with large angular coverage: $10^{\circ}$ to $40^{\circ}(T 1), 30^{\circ}$ to $60^{\circ}(T 2), 50^{\circ}$ to $100^{\circ}(T 3)$, and $90^{\circ}$ to $140^{\circ}$ (T4) [20]. Due to the compact geometry used in the setup, a refined determination of the angle subtended by each pixel of $T 1$ and $T 2$ was done based on the fact that the elastic scattering of ${ }^{9} \mathrm{Li}$ on ${ }^{208} \mathrm{~Pb}$ at energies below the barrier follows the Rutherford scattering formula. For the ${ }^{11} \mathrm{Li}+{ }^{208} \mathrm{~Pb}$ data, the method was applied to the sum of elastic and breakup data. Further details on the setup, data processing, and event selection can be found in Refs. [20,21].

Elastic events were selected in the two-dimensional plot of $\Delta E$ versus $\Delta E+E$ energy spectra for each pixel. A clear identification of the elastic peaks and fragments, both in the ${ }^{9} \mathrm{Li}$ and in the ${ }^{11} \mathrm{Li}$ scattering data, was achieved. Figure 1 illustrates the data obtained for ${ }^{9} \mathrm{Li}$ and ${ }^{11} \mathrm{Li}$ beams scattered on the same $1.45 \mathrm{mg} / \mathrm{cm}^{2}$ thick ${ }^{208} \mathrm{~Pb}$ target and at equivalent c.m. energies. The twodimensional plot for the ${ }^{11} \mathrm{Li}$ scattered data, on the bottom part of Fig. 1, shows the contribution of the elastic channel and the ${ }^{9} \mathrm{Li}$ breakup data. The pixels contributing to the selected angular ring of $(14 \pm 1)^{\circ}$ are displayed on the lefthand side of the figure. The relatively large contribution of the breakup channel observed at this low energy and forward angle is remarkable.

In Fig. 2, we show the two-dimensional plot corresponding to ${ }^{9} \mathrm{Li}+{ }^{208} \mathrm{~Pb}$ scattered data at a c.m. energy of $28.3 \mathrm{MeV}$ for a pixel of telescope 4 centered at $128.2^{\circ}$. It is remarkable that, at this near-threshold energy, one can identify ${ }^{8} \mathrm{Li}$ events likely corresponding to the transfer reaction ${ }^{208} \mathrm{~Pb}\left({ }^{9} \mathrm{Li},{ }^{8} \mathrm{Li}\right){ }^{209} \mathrm{~Pb}(Q=-0.1 \mathrm{MeV})$ and the possible contribution of ${ }^{7} \mathrm{Li}+{ }^{210} \mathrm{~Pb}(Q=3 \mathrm{MeV})$ and $\alpha$ production from transfer or breakup, observed at very high energies [22]. The contribution of these channels, when present, has been removed in order to extract the elastic scattering data. The differential cross section for each detector was calculated by the sum of the counts in each pixel divided by the sum of their solid angles corresponding to a given angular ring.

The measured elastic angular distributions for ${ }^{9} \mathrm{Li}$ and ${ }^{11} \mathrm{Li}$ on ${ }^{208} \mathrm{~Pb}$ are displayed in Fig. 3, relative to the 


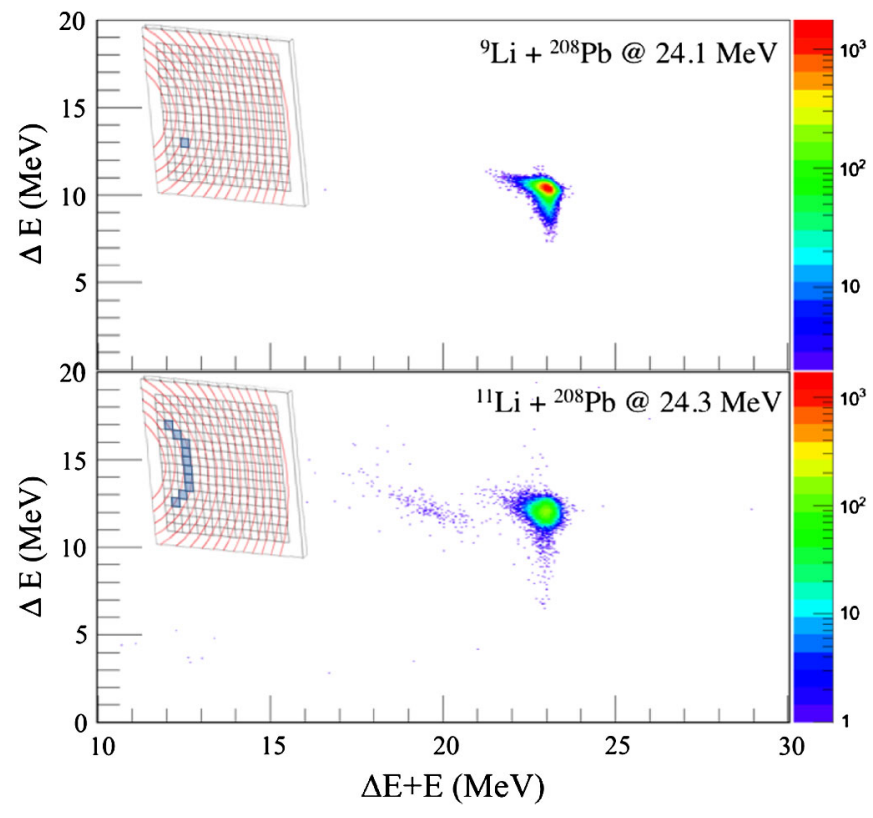

FIG. 1 (color online). Two-dimensional plot of $\Delta E$ versus $\Delta E+E$ in $T 1$ displayed for the pixels illustrated on the left part of the figures. The scattered ${ }^{9} \mathrm{Li}+{ }^{208} \mathrm{~Pb}$ data are shown in the upper part for only one pixel centered at $13.9^{\circ}$. In the bottom figure, the scattered ${ }^{11} \mathrm{Li}+{ }^{208} \mathrm{~Pb}$ data are shown in the angular sector of $(14 \pm 1)^{\circ}$. Contributions from the elastically scattered ${ }^{11} \mathrm{Li}$ and ${ }^{9} \mathrm{Li}$ reakup data are distinctly separated. The data correspond to the same c.m. energy of $23.1 \mathrm{MeV}$.

Rutherford cross section. The upper and lower panels correspond to $E_{\text {c.m. }}=23.1$ and $28.3 \mathrm{MeV}$, respectively. For the lower energy (below the Coulomb barrier), the ${ }^{9} \mathrm{Li}$ data are very close to the Rutherford cross section, whereas, for the higher energy, a Coulomb-nuclear interference maximum starts to be visible at $\theta_{\text {c.m. }} \approx 75^{\circ}$, accompanied by a smooth decrease beyond this angle. This is reminiscent of the Fresnel-type pattern characteristic of the scattering of "normal" nuclei near the Coulomb

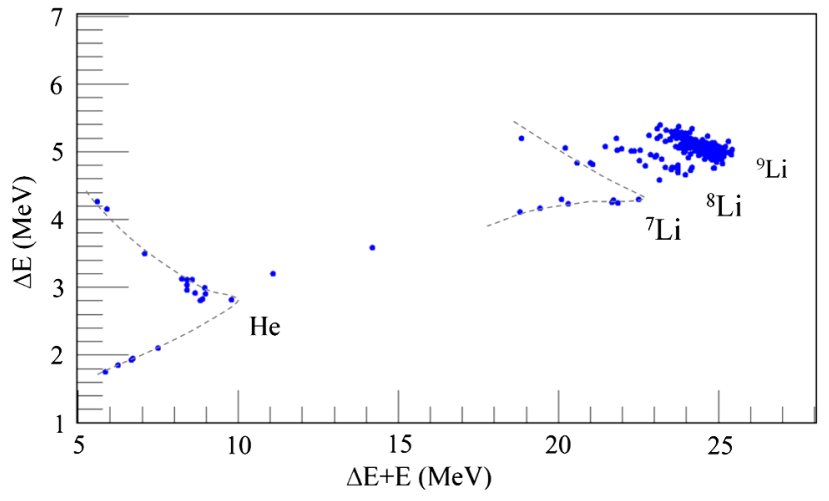

FIG. 2 (color online). Two-dimensional plot of $\Delta E$ versus $\Delta E+E$ for ${ }^{9} \mathrm{Li}$ cattering on a $1.9 \mathrm{mg} / \mathrm{cm}^{2}{ }^{208} \mathrm{~Pb}$ target at $E_{\text {c.m. }}=28.3 \mathrm{MeV}$ for a pixel at $128.2^{\circ}$. Note the presence of breakup channels already at this energy. barrier. On the other hand, the ${ }^{11} \mathrm{Li}$ data show a strong reduction with respect to Rutherford, even at the energy below the barrier. Moreover, the departure from Rutherford scattering at both energies starts at very forward angles. This behavior has been observed previously in the scattering of ${ }^{6} \mathrm{He}$ [23] and ${ }^{11} \mathrm{Be}$ [24], although the observed reduction was not as striking as in the ${ }^{11} \mathrm{Li}$ case.

For a more quantitative understanding of this behavior, we have compared the ${ }^{11} \mathrm{Li}+{ }^{208} \mathrm{~Pb}$ data with four-body CDCC calculations. The CDCC method $[25,26]$ is a generalization of the coupled-channels formalism traditionally used in the analysis of inelastic reactions populating excited states of the projectile or target. In CDCC, the model space is extended so as to include the unbound states of the projectile, thus allowing the evaluation of the projectile breakup and its effect on the elastic scattering. Since the positive energy states form a continuum, a discrete representation in terms of a finite set of square-integrable functions is commonly used. Due to the Borromean structure of the ${ }^{11} \mathrm{Li}$ nucleus, we use here a recent extension of the method appropriate for three-body projectiles (four-body CDCC). This kind of calculation has successfully been used to describe several ${ }^{6} \mathrm{He}$ induced reactions [27-29].

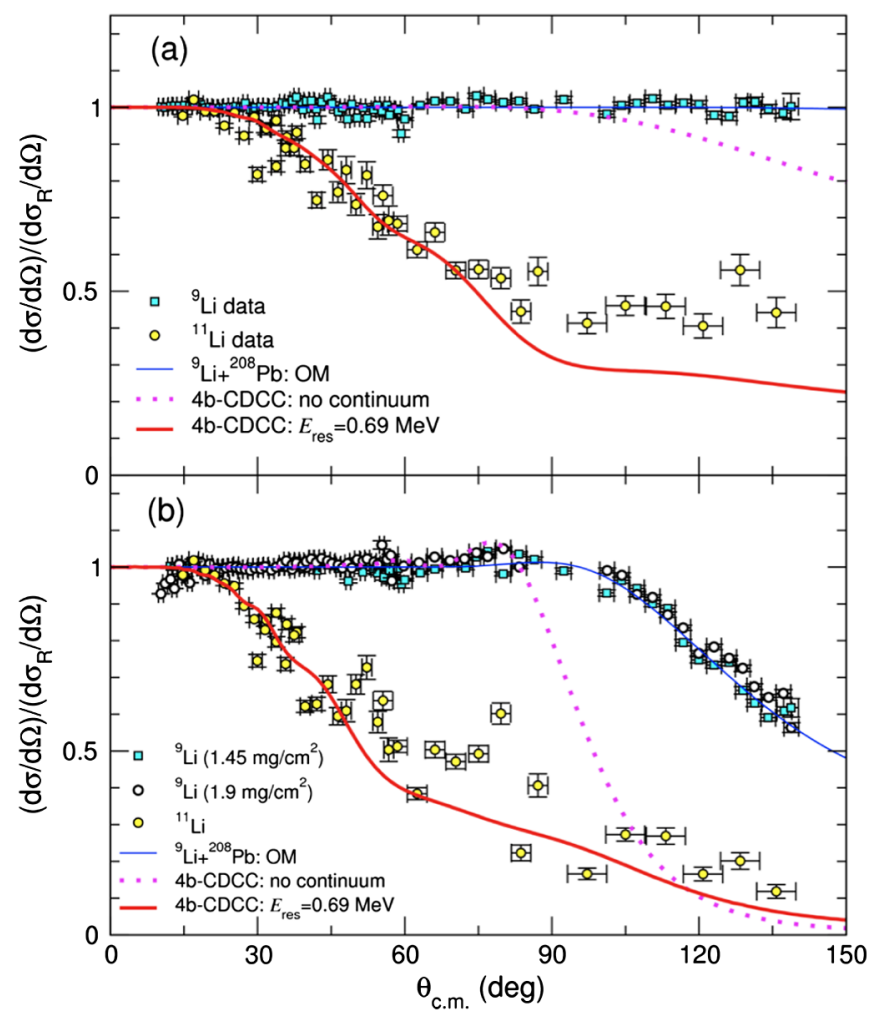

FIG. 3 (color online). Elastic differential cross section of ${ }^{9} \mathrm{Li}$ and ${ }^{11} \mathrm{Li}$ on ${ }^{208} \mathrm{~Pb}$, plotted as a ratio to the Rutherford cross section. In the upper part, it is shown for energies below the barrier, $E_{\text {c.m. }}=23.1 \mathrm{MeV}$, and in the bottom part for $E_{\text {c.m. }}=$ 28.3 MeV. The optical model (OM) calculation for the ${ }^{9} \mathrm{Li}+$ ${ }^{208} \mathrm{~Pb}$ system is also shown in each panel. For details on the fourbody CDCC calculations, see the text. 
To describe the ${ }^{11} \mathrm{Li}$ nucleus, a three-body structure is assumed with an inert and spinless ${ }^{9} \mathrm{Li}$ core surrounded by two valence neutrons. The ${ }^{11} \mathrm{Li}$ states were obtained solving the Schrödinger equation in hyperspherical coordinates. We took the two-body interactions $P 4$ from Ref. [30]. In addition, an effective three-body force was included and adjusted to reproduce the experimental twoneutron separation energy. This gave a ground state wave function with a rms of $3.30 \mathrm{fm}$, assuming a rms of $2.44 \mathrm{fm}$ for the ${ }^{9} \mathrm{Li}$ core [7]. Continuum states were grouped into energy bins, up to a maximum energy of $5 \mathrm{MeV}$, following the average method, recently extended to the three-body continuum [29]. Continuum states with $J_{n n}^{\pi}=0^{+}, 1^{-}, 2^{+}$, and $3^{-}$were included.

The elastic differential cross section for this reaction is very sensitive to the $B(E 1)$ strength close to the threshold and, in particular, to the presence of a dipole resonance. Since the precise location of this resonance has not yet been firmly established, we have adjusted the position of this resonance to the energy that best reproduces the elastic and breakup cross sections. The latter will be presented in a separate work [31]. This gives $E_{\text {res }}=0.69 \mathrm{MeV}$ (i.e., $0.32 \mathrm{MeV}$ above the breakup threshold). To place the dipole resonance at the desired position, the three-body force has been adjusted for the $1^{-}$continuum. This model predicts a large $E 1$ strength at low energies, even larger than that extracted experimentally [14].

For the scattering calculations, the $n+{ }^{208} \mathrm{~Pb}$ and ${ }^{9} \mathrm{Li}+$ ${ }^{208} \mathrm{~Pb}$ interactions are also required. The former was taken from the global parametrization of Koning and Delaroche [32]. The real part of the ${ }^{9} \mathrm{Li}+{ }^{208} \mathrm{~Pb}$ potential was generated microscopically, using the double-folding São Paulo potential [33], with the ${ }^{9} \mathrm{Li}$ matter density from Ref. [7] and the ${ }^{208} \mathrm{~Pb}$ density from a Hartree-Fock calculation. The imaginary part was parametrized using a Woods-Saxon potential for which the parameters $\left(W_{v}, r_{i}, a_{i}\right)$, along with the normalization of the real part $\left(N_{r}\right)$, were adjusted in order to reproduce the present ${ }^{9} \mathrm{Li}$ elastic data at $E_{\mathrm{c} . \mathrm{m} .}=$ $28.3 \mathrm{MeV}$, giving rise to the parameters $N_{r}=1.0, W_{v}=$ $46.5 \mathrm{MeV}, r_{i}=1.33 \mathrm{fm}$, and $a_{i}=0.47 \mathrm{fm}$. The optical model calculations for ${ }^{9} \mathrm{Li}+{ }^{208} \mathrm{~Pb}$ are compared with the data in Fig. 3 (for further details about the procedure, see Ref. [20]).

The CDCC coupled differential equations were solved with the code FRESCO [34], including both nuclear and Coulomb couplings between the target and the projectile to all orders. The four-body CDCC calculations are compared with the ${ }^{11} \mathrm{Li}+{ }^{208} \mathrm{~Pb}$ data in Fig. 3. To illustrate the effect of the coupling to the breakup channels, we have included also the calculation in which these couplings are omitted (dotted line). This four-body CDCC calculation without coupling to the continuum follows the ${ }^{9} \mathrm{Li}$ data up to $\sim 90^{\circ}$ but then decreases faster than the ${ }^{9} \mathrm{Li}$ data beyond this angle, as illustrated in the lower part of Fig. 3. This reduction is due to the absorptive effect arising from the interaction of the halo neutrons with the target and also to the extended size of the ${ }^{11} \mathrm{Li}$ ground state. This diminution, however, is found to be insufficient to explain the ${ }^{11} \mathrm{Li}$ data. Including the coupling to the continuum produces an additional reduction, and the resulting angular distribution is found to describe very well the data at both energies. At $E_{\text {lab }}=24.3 \mathrm{MeV}$, the CDCC calculations underestimate the data at the largest angles. Inclusion of continuum states of even larger angular momenta (e.g., $J_{n n}^{\pi}=4^{+}$) could increase the calculated elastic cross sections even further. Our CDCC calculations indicate that the main interactions responsible for the reduction of the elastic cross section and the subsequent disappearance of the Coulomb-nuclear interference peak are the dipole Coulomb couplings. This special behavior of ${ }^{11} \mathrm{Li}$ has been associated with the effect of Coulomb dipole polarizability [15]. The weakly bound ${ }^{11} \mathrm{Li}$, in the strong Coulomb field of the target, gets distorted and eventually breaks up. This reduces the elastic cross sections similarly below $\left(E_{\text {c.m. }}=23.1 \mathrm{MeV}\right)$ and at the barrier top $\left(E_{\text {c.m. }}=28.3 \mathrm{MeV}\right)$, where the absence of the Coulomb-nuclear interference peak is extraordinary.

In summary, the first measurement of the elastic scattering of the halo nucleus ${ }^{11} \mathrm{Li}$ and its core ${ }^{9} \mathrm{Li}$ on ${ }^{208} \mathrm{~Pb}$ at energies below and on top of the Coulomb barrier is presented. The ${ }^{9} \mathrm{Li}+{ }^{208} \mathrm{~Pb}$ scattering data follow the expected behavior for well-bound nuclei, obeying the Rutherford formula below the barrier and exhibiting a Fresnel-like diffraction pattern above the barrier. On the other hand, the ${ }^{11} \mathrm{Li}+{ }^{208} \mathrm{~Pb}$ elastic cross section departs significantly from the standard behavior of a well-bound nucleus such as its core ${ }^{9} \mathrm{Li}$, showing a strong reduction with respect to the Rutherford cross section both below and around the Coulomb barrier. Four-body CDCC calculations, using a three-body model of ${ }^{11} \mathrm{Li}$ and including Coulomb and nuclear couplings to all orders, reproduce satisfactorily the experimental elastic angular distributions. The reduction of the cross section with respect to the Rutherford scattering is attributed to the strong dipole coupling between the ground and the continuum states in ${ }^{11} \mathrm{Li}$. The presence of a low-lying dipole resonance, close to the breakup threshold, is found to improve the agreement with the data.

This work has been partially supported by Spanish National Projects No. FPA2009-07387, No. FPA200907653, No. FPA2009-08848, and No. FPA2010-22131C02-01, by the Consolider-Ingenio 2010 Program CPAN (CSD2007-00042), and by the U.K. Science and Technology Facilities Council through Grant No. EP/ D060575/1. M.C. acknowledges the support of the CSIC-UCR and FCT Grant No. ISFL-2-275.

*mj.borge@csic.es

[1] E. Rutherford, Philos. Mag. 21, 669 (1911).

[2] H. Geiger and E. Marsden, Proc. R. Soc. A 82, 495 (1909). 
[3] P. G. Hansen and B. Jonson, Europhys. Lett. 4, 409 (1987).

[4] I. Tanihata, H. Hamagaki, O. Hashimoto, Y. Shida, N. Yoshikawa, K. Sugimoto, O. Yamakawa, T. Kobayashi, and N. Takahashi, Phys. Rev. Lett. 55, 2676 (1985).

[5] A. M. Poskanzer, R. McPherson, R. A. Esterlund, and P. L. Reeder, Phys. Rev. 152, 995 (1966).

[6] M. Smith et al., Phys. Rev. Lett. 101, 202501 (2008).

[7] A. V. Dobrovolsky et al., Nucl. Phys. A766, 1 (2006).

[8] J.H. Kelley, E. Kwan, J.E. Purcell, C. G. Sheu, and H. R. Weller, Nucl. Phys. A880, 88 (2012).

[9] A. Cobis, D. V. Fedorov, and A. S. Jensen, Phys. Rev. C 58, 1403 (1998).

[10] I. J. Thompson, B. V. Danilin, V. D. Efros, M. V. Zhukov, J. S. Vaagen, and The Russian-Nordic-British Theory collaboration, J. Phys. G 24, 1505 (1998).

[11] K. Ikeda, Nucl. Phys. A538, 355 (1992).

[12] E. Garrido, D. V. Fedorov, and A. S. Jensen, Nucl. Phys. A708, 277 (2002).

[13] E. C. Pinilla, P. Descouvemont, and D. Baye, Phys. Rev. C 85, 054610 (2012).

[14] T. Nakamura et al., Phys. Rev. Lett. 96, 252502 (2006).

[15] M. V. Andrés and J. Gómez-Camacho, Phys. Rev. Lett. 82, 1387 (1999).

[16] N. K. Skobelev, S. M. Lukyanov, Y.E. Penionzhkevich, S.P. Tretyakova, C. Borcea, V.E. Zhuchko, V.A. Gorshkov, K. O. Terenetsky, V.P. Verbitsky, and Y.A. Pozdnyakov, Z. Phys. A 341, 315 (1992).

[17] A. M. Vinodkumar, W. Loveland, P. H. Sprunger, L. Prisbrey, M. Trinczek, M. Dombsky, P. Machule, J. J. Kolata, and A. Roberts, Phys. Rev. C 80, 054609 (2009).

[18] G. C. Ball, L. Buchmann, B. Davids, R. Kanungo, C. Ruiz, and C. E. Svensson, J. Phys. G 38, 024003 (2011).

[19] O. Tengblad, U.C. Bergmann, L. M. Fraile, H.O.U. Fynbo, and S. Walsh, Nucl. Instrum. Methods Phys. Res., Sect. A 525, 458 (2004).
[20] M. Cubero et al., Eur. Phys. J. Web Conf. 17, 16002 (2011).

[21] M. J. G. Borge et al., J. Phys. Conf. Series 381, 012085 (2012).

[22] P. D. Zecher et al., Phys. Rev. C 57, 959 (1998).

[23] E. F. Aguilera et al., Phys. Rev. C 63, 061603 (2001); O. R. Kakuee et al., Nucl. Phys. A728, 339 (2003); A765, 294 (2006).

[24] A. Di Pietro et al., Phys. Rev. Lett. 105, 022701 (2010).

[25] M. Kamimura, M. Yahiro, Y. Iseri, Y. Sakuragi, H. Kameyama, and M. Kawai, Prog. Theor. Phys. Suppl. 89, 1 (1986).

[26] N. Austern, Y. Iseri, M. Kamimura, M. Kawai, G. Rawitscher, and M. Yahiro, Phys. Rep. 154, 125 (1987).

[27] T. Matsumoto, E. Hiyama, K. Ogata, Y. Iseri, M. Kamimura, S. Chiba, and M. Yahiro, Phys. Rev. C 70, 061601 (2004); T. Matsumoto, T. Egami, K. Ogata, Y. Iseri, M. Kamimura, and M. Yahiro, Phys. Rev. C 73, 051602 (2006).

[28] M. Rodríguez-Gallardo, J. M. Arias, J. Gómez-Camacho, R. C. Johnson, A. M. Moro, I.J. Thompson, and J. A. Tostevin, Phys. Rev. C 77, 064609 (2008).

[29] M. Rodríguez-Gallardo, J. M. Arias, J. Gómez-Camacho, A. M. Moro, I. J. Thompson, and J. A. Tostevin, Phys. Rev. C 80, 051601(R) (2009).

[30] I. J. Thompson and M. V. Zhukov, Phys. Rev. C 49, 1904 (1994).

[31] J.P. Fernández-García et al. (to be published).

[32] A. J. Koning and J. P. Delaroche, Nucl. Phys. A713, 231 (2003).

[33] L. C. Chamon, B. V. Carlson, L. R. Gasques, D. Pereira, C. De Conti, M. A. G. Alvarez, M. S. Hussein, M. A. Cândido Ribeiro, E. S. Rossi, Jr., and C. P. Silva, Phys. Rev. C 66, 014610 (2002).

[34] I. J. Thompson, Comput. Phys. Rep. 7, 167 (1988). 